\title{
Conhecimento sobre o exame citológico pelas profissionais de uma Instituição de Ensino Superior no município de Patos-PB
}

\author{
Cytological examination knowledge for professionals of a higher education \\ Institution in the city of Patos-PB
}

\author{
Niedja Alves de Araujo ${ }^{1}$, Maria Luisa Souto Porto ${ }^{2}$
}

\author{
${ }^{1}$ Discente do curso de Biomedicina das Faculdades Integradas de Patos. \\ ${ }^{2}$ Mestre em Ciências da Saúde (UFRN). Hospital Universitário Alcides Carneiro - \\ Universidade Federal de Campina Grande (PB).
}

\begin{abstract}
Resumo
Introdução: o câncer do colo do útero é um dos tipos de câncer mais comuns entre as mulheres e tem um alto índice de mortalidade, mas se for detectado precocemente através da realização do exame preventivo Papanicolaou, torna-se a melhor forma de se chegar ao restabelecimento da saúde. Objetivo: a pesquisa teve como objetivo identificar o conhecimento das funcionárias de uma Instituição de Ensino Superior sobre o exame preventivo do câncer do colo do útero. Metodologia: o presente estudo trata-se de uma pesquisa quantitativa, onde participaram 40 mulheres que fazem parte do quadro de funcionárias da Instituição, com idade de 19 a 69 anos. A pesquisa foi realizada nas Faculdades Integradas de Patos - FIP localizada na cidade de Patos no Estado da Paraíba. Utilizou-se um questionário adaptado pelos pesquisadores de respostas dicotômicas (SIM/NÃO) e alternativas contendo 15 perguntas. Resultados: a pesquisa demonstra que o nível de conhecimento das funcionárias sobre o exame citológico é de $97 \%$ ( $n=39$ ). Foi verificado ainda, que $92 \%(n=37)$ funcionárias sabem qual a finalidade do exame e $90 \%(n=36)$ conhecem o procedimento de realização do exame. Conclusão: diante dos resultados apresentados, conclui-se que na população estudada a maioria das mulheres conhecem o exame Papanicolaou, sabem qual a finalidade e o procedimento do mesmo. Entretanto, ainda se faz necessário o desenvolvimento de programas de ações voltadas para a educação em saúde. É preciso também, haver investimentos na saúde da mulher dentro da própria Instituição de Ensino e associar campanhas do exame preventivo Papanicolaou com atividades educativas.

Palavras-chave: : Câncer do colo do útero. Conhecimento. Mulheres. Prevenção.
\end{abstract}

\begin{abstract}
Background: cervical cancer is one of the types of cancer more common among women and has a high rate of mortality, but if it is detected early through Papanicolaou preventive examination, it is the best way to get to the restoration of health. Goal: The research aimed to identify the knowledge of the employees of an institution of higher education the preventive examination of cervical cancer. Methodology: the present study it is a quantitative research, attended by 40 women who are part of the staff of the institution, aged from 19 to 69 years. The survey was conducted in the Faculdades Integradas de Patos-FIP located in the city of Patos in the State of Paraiba. It was used a questionnaire adapted by researchers dichotomous responses (YES/NO) and alternatives containing 15 questions. Results: the research demonstrates that the level of knowledge of the employees on the cytological examination is $97 \%(n=39)$. Has been verified yet, that $92 \%(n=37)$ employees know what the purpose of the examination and $90 \%(n=36)$ know the procedure of realization of examination. Conclusion: before the results presented it is concluded that in the studied population most women know the Papanicolaou smear examination, know what the purpose and procedure of the same. However, still it is necessary to the development of programs of actions geared toward health education. You also need to, there are investments in women's health within the educational institution and associate exam Papaicolaou preventive campaigns with educational activities. Keywords: Cancer of the cervix. Knowledge. Women. Prevention.
\end{abstract}

\section{INTRODUÇÃO}

O Câncer do colo do útero é uma neoplasia maligna onde ocorrem mudanças intraepiteliais e de forma lenta pode se transformar num processo invasivo1. É um dos tipos mais comuns de câncer entre as mulheres. Aproximadamente 275 mil mulheres no mundo morrem devido a esse tipo de câncer. Sua incidência maior é registrada nos países menos desenvolvidos. A incidência é geralmente em mulheres de 20 a 29 anos e um pico etário entre 50 e 60 anos $^{2}$.

Correspondência / Correspondence: Niedja Alves de Araujo. Faculdades Integradas de Patos - FIP. Rua Horácio Nóbrega, s/n - Belo Horizonte. 58704-000 Patos - PB - Brasil. Tel.: (83) 8123-2201.

Email: niedjaalves9130@gmail.com
No Brasil, conforme as estimativas do Instituto Nacional do Câncer (INCA) apontam que no ano de 2012 ocorreria em torno de 17.000 novos casos, sendo a região Nordeste a segunda região mais incidente desse tipo de câncer, ficando atrás apenas da região Norte 2 .

No ano de 1988, foi adotada no Brasil uma norma da Organização Mundial de Saúde (OMS) ao qual propunha que as mulheres de 25 a 60 anos realizassem o exame citológico pelo menos uma vez a cada ano para que assim pudesse haver um controle do câncer cérvico-uterino $^{3}$. Hoje, o exame popularmente conhecido como Papanicolaou é o exame mais utilizado para diagnosticar precocemente a doença $a^{2}$. O exame previne o câncer do 
colo do útero e tem uma grande importância para as mulheres, além de desempenhar um importante papel na detecção precoce de lesões pré-invasivas, fazendo com que o número de mortes diminua ${ }^{4}$. No Brasil, muitas mulheres só procuram o serviço de saúde para fazer o exame de prevenção do câncer do colo do útero quando estão apresentando algum problema de saúde. Em consequência, a maioria dos casos quando diagnosticados, mostram que a doença já está avançada, diminuindo assim, a chance de cura 5 .

O fator principal que leva ao desenvolvimento de lesões intraepiteliais e ao câncer do colo do útero é a infecção pelo papilomavírus humano (HPV). Mas não só a infecção por este vírus faz com que surja o câncer, é necessário uma série de fatores que juntos facilitam a doença, como por exemplo, a idade, o tabagismo, a imunidade, a genética, o comportamento sexual, dentre outros $^{2}$.

Percebendo-se que o câncer do colo do útero apresenta uma alta incidência e um grande número de mortalidade, mas que a detecção precoce é a melhor forma de se chegar à cura, buscou-se com esse projeto identificar o conhecimento das mulheres que são funcionárias de uma instituição de ensino superior sobre a prevenção contra esse tipo de câncer.

\section{MATERIAIS E MÉTODOS}

O presente estudo trata-se de uma pesquisa quantitativa, onde através de um questionário foi verificado o conhecimento de mulheres a respeito do exame citológico. A pesquisa foi realizada nas Faculdades Integradas de Patos - FIP, localizada na cidade de Patos no estado da Paraíba. A população foi formada por 40 muIheres que são funcionárias da instituição de ensino superior participante da pesquisa.

Os critérios de inclusão foram mulheres que fizessem parte do quadro de funcionárias (não docentes) das FIP, maiores de 18 anos e que tivessem assinado o Termo de Consentimento Livre e Esclarecido - TCLE. Os critérios de exclusão foram funcionárias que estudassem em algum curso da área de saúde ou que já tivessem participado de palestras sobre o exame citológico, como também, funcionárias que estavam afastadas na época da entrevista ou que se recusaram a participar como voluntária.

A coleta de dados foi feita através da aplicação de um questionário adaptado pelos pesquisadores de respostas dicotômicas (SIM/NÃO) e alternativas contendo 15 perguntas. Os dados da amostra foram tabulados, analisados exposto em formas de gráficos sendo utilizado o Programa da Microsoft (Excel 2007). Os resultados foram apresentados de forma descritiva para melhor compreensão dos dados expostos em gráficos.

A realização deste estudo considerou a Resolução no 196/96 do Conselho Nacional de Saúde que rege sobre a ética da pesquisa envolvendo seres humanos direta ou indiretamente, assegurando a garantia de que a privacidade do sujeito da pesquisa será preservada. Este projeto foi submetido ao Comitê de Ética em Pesquisa das Faculdades Integradas de Patos. Após a concessão de sua aprovação, todos os sujeitos envolvidos na pesquisa assinaram ao TCLE, que foi impresso em duas vias, uma para o pesquisado e outra para o pesquisador. A preservação da privacidade dos sujeitos foi garantida por meio do Termo de Compromisso do Pesquisador 6 .

\section{RESULTADOS}

Ao serem realizadas as pesquisas com as funcionárias através do questionário, seguindo o direcionamento do percurso metodológico, foi possível a caracterização da amostra, sendo descritas a seguir no Figura 1.

Figura 1. Dados Sócio-demográficos das funcionárias das Faculdades Integradas de Patos - FIP no município de Patos-PB.

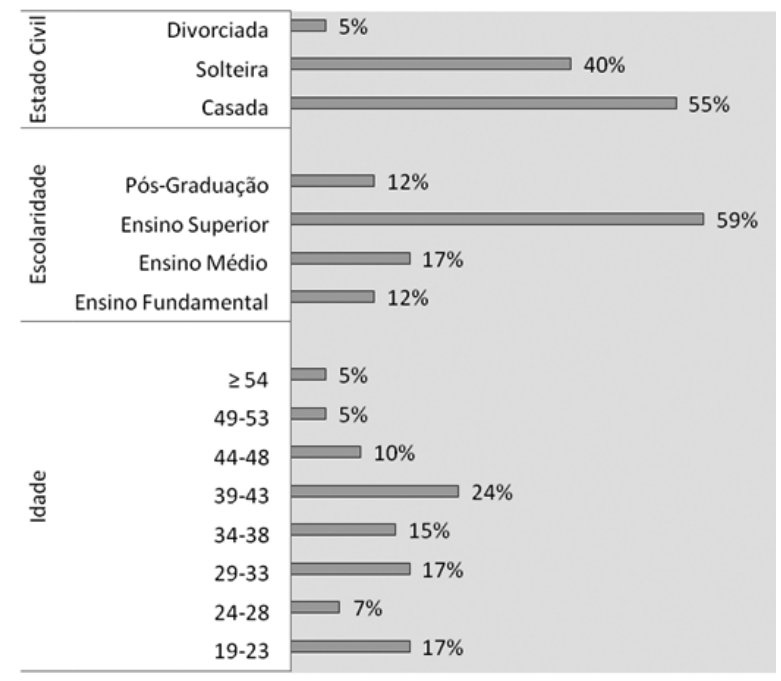

Conforme apresentado no Figura 1, a análise dos dados relativos às características das funcionárias, demonstrou que a faixa etária predominante incidiu sobre 39 a 43 anos, que corresponde a $24 \%(n=9)$ funcionárias e a faixa etária que menos predominou foi de 49 a 53 anos, que corresponde a $5 \%(n=2)$ funcionárias e $\geq 54$ anos, que corresponde também a $5 \%(n=2)$ funcionárias. Nos dados referente à escolaridade, foi verificado que $12 \%(n=05)$ concluíram o ensino fundamental, $17 \%(n=07)$ o ensino médio, $59 \%(n=23)$ o ensino superior e $12 \%(n=05)$ eram pós-graduadas. Observou-se que a predominância nos dados sobre escolaridade é de mulheres que possuem o ensino superior. Quanto ao estado civil foi analisado que $55 \%(n=22)$ das funcionárias eram casadas, $40 \% \quad(n=16)$ solteiras e $5 \%(n=2)$ divorciadas.

A pesquisa demonstrou que os maiores percentuais das funcionárias conhecem o exame citológico, sabem qual a finalidade e o procedimento de realização do mesmo, conforme é demonstrado no Figura 2. 
Figura 2. Conhecimento, finalidade e procedimento do exame citológico Papanicolaou.

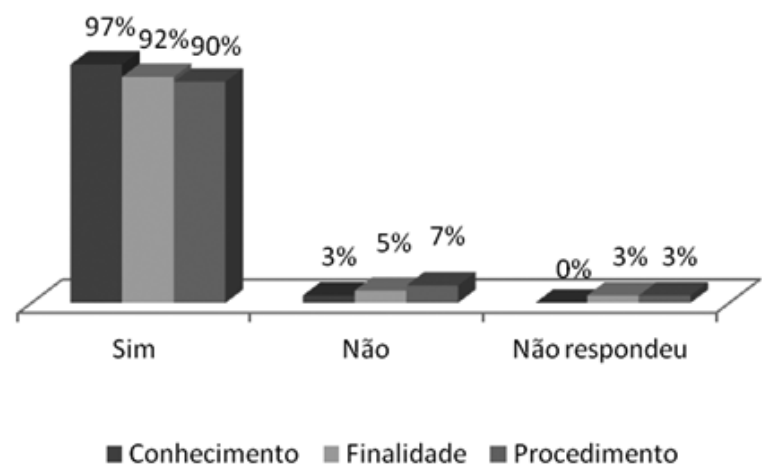

Conforme apresentado na Figura 2, a pesquisa demonstra que o nível de conhecimento das funcionárias sobre o exame citológico é de $97 \%(n=39)$. Foi verificado ainda, que $92 \%(n=37)$ funcionárias sabem qual a finalidade do exame e $90 \%(n=36)$ conhecem o procedimento de realização do exame.

Figura 3. Local onde é realizado o exame citológico Papanicolaou.

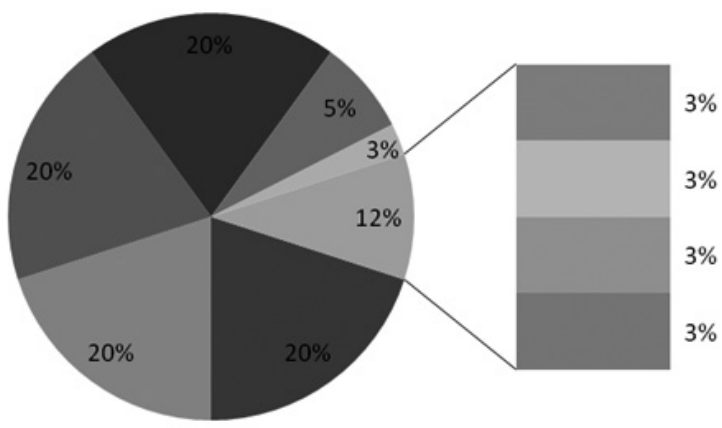

\begin{tabular}{|c|c|}
\hline aClínica & —Clínica e PSF \\
\hline — Laboratório, Clínica e PSF & $\square$ PSF \\
\hline Laboratório e PSF & Laboratório, Clínica, Hospital e PSF \\
\hline Eospital & NR \\
\hline Não sei & - Laboratório \\
\hline
\end{tabular}

A Figura 3, mostra que apenas $3 \%(n=1)$ funcionárias não sabem onde pode ser realizado o exame e $3 \%(n=1)$ funcionárias não respondeu.

Os cuidados prévios necessários para a realização do exame de Papanicolaou é um fator condicionante para o desfecho favorável na pesquisa. Na Figura 4, a pesquisa demonstra que poucas funcionárias não sabem ou não responderam quais os cuidados necessários antes do exame, isso mostra mais uma vez, o conhecimento que elas tem sobre o exame citológico do Papanicolaou.
Figura 4. Cuidados prévios necessários para a realização do exame Papanicolaou.

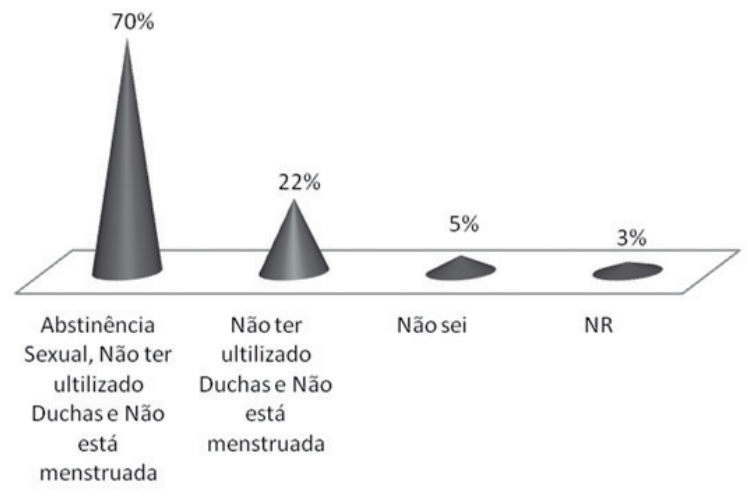

Conforme apresentado a Figura 4, 70\% (n=28) funcionárias sabem todos os cuidados prévios necessários para a realização do exame, $22 \%(n=9)$ funcionárias desconhecem que devem está em abstinência sexual, que é um dos cuidados mais necessários que se deve ter antes de realizar o exame citológico, $5 \%(n=2)$ funcionárias não sabem quais os cuidados prévios e apenas $3 \%$ $(n=1)$ não respondeu.

Os interferentes que levam as mulheres a não realizar o exame preventivo são um dos fatores mais relevantes da pesquisa, como mostra a Figura 5:

Figura 5. Interferentes que levam a não realização do exame citológico Papanicolaou.

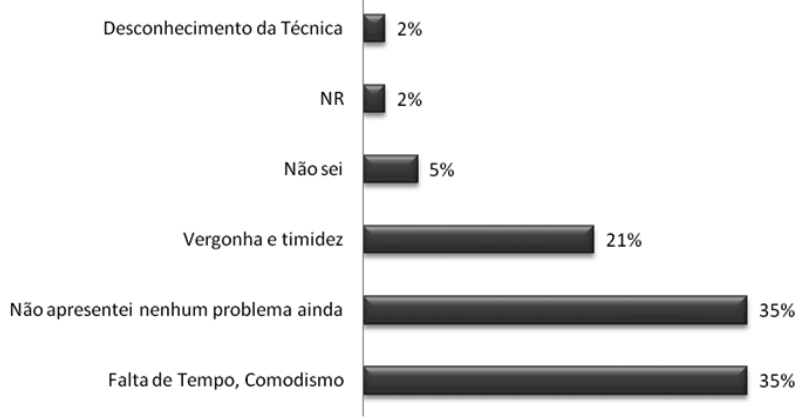

Ao analisar a Figura 5, foi identificado que os interferentes que são mais predominantes na pesquisa são Falta de tempo, comodismo, com 35\% ( $n=14)$ funcionárias e "Não apresentei nenhum problema ainda", também com $35 \%(n=14)$ funcionárias, seguidos por Vergonha e timidez $21 \%(n=8)$ funcionárias. Foi observado que um dos interferentes mais relevantes para estas funcionárias era mais pelo aspecto curativo do que preventivo. Onde mostra que uma grande parte das funcionárias só faz o exame preventivo quando apresentam algum problema e isso se deve à falta de informação e, consequentemente, à falta de conhecimento a respeito desta temá- 
tica. Os interferentes que menos predominaram na pesquisa foram $5 \%(n=2)$ funcionárias que não sabiam quais os interferentes, $2 \%(n=1)$ funcionárias que desconheciam a técnica e $2 \%(n=1)$ funcionárias não responderam. Foi observado, através do questionário, que $97 \%$ ( $n=39)$ funcionárias responderam que o esposo/companheiro não interfere na realização do exame e 3\% (n=1) funcionárias não responderam. Através dos dados, reforça-se a importância do profissional de saúde fazer parte do desenvolvimento de estratégias e programas de conhecimento da saúde para a sociedade.

\section{DISCUSSÃO}

Estudos relacionados apontam que o índice de conhecimento da população feminina sobre o exame citológico do Papanicolaou e instruções relacionadas a este exame preventivo tendem a variar quanto a alguns fatores sócio-demográficos, como foram descritos neste estudo representados nas Figuras 1 e 2, mostrando algumas variáveis sócio-demográficas analisadas, tais como: escolaridade, idade, estado civil, caracterizando assim a amostra do estudo, seguido pela correlação dos conhecimentos sobre o exame preventivo das participantes.

De acordo com um estudo realizado por Torres e Brito (2006) o conhecimento das mulheres entre 25 e 50 anos, com nível de escolaridade variável, sobre o exame preventivo Papanicolaou, apontou que 32,14\% já tinham ouvido falar no exame citológico, enquanto que $21,43 \%$ nunca ouviram falar do referido exame ${ }^{7}$. Concomitante a este estudo mulheres da área urbana, de classe média, aquelas com maior escolaridade, as solteiras, e as que usam algum método contraceptivo, apresentaram maior adequação do conhecimento sobre o exame. Visto que há o maior acesso às informações sobre o exame e mais oportunidades para fazê-lo. No caso das mulheres solteiras, o maior conhecimento devido ao fato de que elas procuram mais por orientação médica no sentido de evitar gravidez não planejadå.

A dificuldade de acesso ao serviço de saúde também foi abordado neste estudo onde foi questionado o local ideal para realização do Papanicolaou, visto na Figura 3. Segundo Domingos e colaboradores (2007) as mulheres relatam que a maior dificuldade ao acesso do serviço de saúde são os horários de agendamento igual ao horário de trabalho, demora ou mau atendimento, conhecimento ineficaz sobre os locais e profissionais adequados à realização do exame ${ }^{9}$. Silva e colaboradores (2006) abordam a necessidade dos profissionais e gestores de saúde estarem atentos às dificuldades de acesso e de utilização dos serviços pela população, especialmente sob modalidade de atenção que pressupõe ênfase na promoção da saúde e na prevenção de agravos, como o da Saúde da Família ${ }^{10}$.

As instruções médicas para realização da coleta do citológico devem ser seguidas pelas pacientes para se obter uma boa qualidade na realização do exame e liberação de um prognóstico com clareza pelo citologista, visto na Figura 4, a pesquisa afirmou que a maior parte das entrevistadas desconhecia a abstinência sexual como conduta a ser seguida antes da realização da coleta do exame citológico.

Neste sentido, a questão do desconhecimento sobre a finalidade e procedimento das condutas necessárias para realização do exame revela uma característica nas populações mais carentes que influência de modo negativo no prognóstico de vários tipos de câncer ${ }^{8}$. Ressaltando que algumas mulheres podem ter informações equivocadas e, por vezes, não distinguir apropriadamente a coleta de material e procedimentos necessários para o exame preventivo ${ }^{11}$.

O câncer de colo de útero não apresenta sintomas na sua fase inicial, por isso a mulher deve fazer o exame citopatológico rotineiramente antes que apareçam os primeiros sintomas, pois se o diagnóstico e o tratamento forem precoces a chance de cura pode ser maior $^{12}$. As entrevistadas relataram em sua grande parte que a não realização do preventivo estava associada ao não aparecimento de alterações ginecológicas, comodismo, falta de tempo, vergonha e medo, visto na Figura 5. A motivação das entrevistadas para realizar o exame está vinculada ao aparecimento de sintomas e a preocupação com sua condição de sua saúde, e também no fato de reconhecerem que seus companheiros podem ser risco potencial para o contágio com $\mathrm{DST}^{13}$.

\section{CONCLUSÃO}

O exame citológico do Papanicolaou é uma forma de diagnóstico eficaz para prevenção de câncer do colo do útero, doença que têm sido responsável pelo aumento da taxa de mortalidade de mulheres nas diversas populações. Embora o exame do Papanicolaou seja acessível no Sistema Único de Saúde (SUS), nota-se uma grande evasão das pacientes na realização deste exame pelas mais diversas explicações.

Os dados obtidos de acordo com a análise das entrevistas colocaram em evidência o conhecimento que as funcionárias tinham em relação ao exame preventivo e os principais interferentes na realização do mesmo.

Foi verificado que ainda existem funcionárias, embora em uma pequena porcentagem, que nunca ouviu falar do exame Papanicolaou, bem como sua finalidade e o seu procedimento. Muitos fatores podem ser destacados como determinantes deste resultado, como a falta de conhecimento, comodismo e a falta de tempo. Por um lado, dessa forma, nós como profissionais de saúde, temos o dever de garantir a adesão dessas mulheres a programas que levem ao conhecimento sobre o exame preventivo do câncer do colo do útero. Por outro lado, observou-se que a maioria das funcionárias possui conhecimento sobre o exame. Embora a consulta médica tenha sido relatada como a principal responsável pela divulgação do exame, faz-se necessário à implementação de programas educativos que contenham informações sobre o exame dentro da própria Instituição de Ensino. 
Sugere-se que a Instituição de Ensino Superior procure melhorar as informações e estratégias utilizadas para que as informações sobre o exame de Papanicolaou possa chegar de forma clara e concisa às funcionárias, enfatizando as vantagens e benefícios para sua saúde e a necessidade da sua realização periódica.

\section{REFERÊNCIAS}

1. THUN, M. J. et al. The global burden of câncer: priorities for prevention. Carcinogenesis, Oxford, v. 31, n. 1, p. 100-110, 2010.

2. BRASIL. MINISTÉRIO DA SAÚDE. INSTITUTO NACIONAL DO CÂNCER (INCA). Estimativas 2012: Incidência de Câncer no Brasil. Rio de Janeiro: INCA, 2011. Disponível em: <http://www1.inca.gov.br/estimativa/2012/index.asp?ID=5>. Acesso em: 21 mai. 2013.

3. ZEFERINO, L. C. et al. Screening da neoplasia cervical. J. Bras. Ginecol., Rio de Janeiro, v. 106, n. 11-12, p. 415-419, 1996.

4. PELLOSO, S. M.; CARVALHO, M. D. B.; HIGARASHI, I. H. Conhecimento das mulheres sobre o câncer cérvico-uterino. Acta Sci., Health Sci., Maringá, v. 26, n. 2, p. 319-324, 2004.

5. VALE, D. B. A. P. et al. Avaliação do rastreamento do câncer do colo do útero na Estratégia Saúde da Família no Município de Amparo, São Paulo, Brasil. Cad. Saúde Pública, Rio de Janeiro, v. 26, n. 2, p. 383-390, 2010.

6. BRASIL. Ministério da Saúde. Conselho Nacional de Saúde. Diretrizes e normas regulamentadoras de pesquisas envolvendo seres humanos. Disponível em: <http://conselho.saude.gov.br/resolucoes/reso_96. htm>. Acesso em: 06 de jun. 2013.
7. TORRES, L. C.; BRITO, C. M. S. Perspectivas das mulheres na realização da citologia oncótica. 2006. 20 f. Trabalho de Conclusão de Curso (Graduação em Enfermagem) - Faculdade de Odontologia e Enfermagem - FACOE, Universidade Estadual do Piauí, Parnaíba, 2006.

8. CASTRO, L. F. Exame Papanicolau: o conhecimento das mulheres sobre o preventivo e a estratégia do PSF no combate ao Câncer de Colo de Útero. 2010. 19 f. Trabalho de Conclusão de Curso (Especialização em atenção básica em saúde da família) - Universidade Federal de Minas Gerais, Uberaba, 2010.

9. DOMINGOS, A. C. P. et al. Câncer do colo do útero: comportamento preventivo de auto-cuidado à saúde. Ciênc., Cuid. Saúde, Maringá, v. 6, n. 2, p. 397-403, 2007.

10. SILVA, D. W. S. et al. Cobertura e fatores associados com a realização do exame Papanicolaou em município do Sul do Brasil. Rev. Bras. Ginecol. Obstet., Rio de Janeiro, v. 28, n. 1, p. 24-31, 2006.

11. OLIVEIRA, S. L.; ALMEIDA, A. C. H. A percepção das Mulheres Frente ao Exame de Papanicolau: da observação ao entendimento. Cogitare Enferm., Curitiba, v. 14, n. 3, p. 518-526, 2009.

12. INSTITUTO NACIONAL DO CÂNCER (INCA). Estimativas 2010: Incidência de Câncer no Brasil. Rio de Janeiro, INCA, 2009. Disponível em: <http://www.inca.gov.br/estimativa/2010/estimativa20091201.pdf>. Acesso em: 21 mai. 2013.

13. DUAVY, L. M. et al. A percepção da mulher sobre o exame preventivo do câncer cérvico-uterino: estudo de caso. Ciênc. Saúde Coletiva, Rio de Janeiro, v. 12, n. 3, p. 733-742, 2007.

Submetido em 06.06.2013;

Aceito em 27.09.2013. 